(Aus der Universitätsklinik für Geisteskranke, Moskau [Dir.: Prof. Dr. P. B. Gannuschkin].)

\title{
Zur Lehre von der Paranoia.
}

\author{
Von \\ T. A. Geier, \\ Oberarzt der Klinik.
}

(Eingegangen am 22. Mai 1922.)

Schon a priori war es anzunehmen, daß die Eigenart unserer jetzigen Zeitverhältnisse: der Umsturz der Revolution, der Um- und Wiederaufbau unseres gesamten alltäglichen Lebens, nicht ohne Einfluß auf die Zahl und Form der seelischen Erkrankungen bleiben konnte; und in der Tat vermerken wir bei unserem klinischèn Gesamtmaterial, da $B$ die jüngste Zeit nicht nur eine Zunahme manisch-depressiver Erkrankungen, insbesondere depressiver Färbung, nicht nur eine erhöhte Frequenz reaktiver Irreseinsformen, sondern auch, und hier das. Wichtigste, ein ungemein häufiges Zumvorscheinkommen sogenannter paranoischer Veranlagungen, gewiß nicht ohne Zusammenhang mit den stürmischen Perturbationen im Gesamtleben unseres Volkes, aufzuweisen hat.

Die Jahre 1919-1921 brachten in unsere Klinik nicht weniger als zwölf paranoische Kranke: zwölf Fälle echter Kraepelinscher Paranoia, während man früher jahrelang keinen einzigen derartigen Fall ausfindig machen konnte. Sämtliche Fälle befanden sich in Behandlung und Beobachtung Molodenkows (Intern der Klinik) und werden demnächst seinerseits, ausführlich beschrieben und besprochen, veröffentlicht. In einer vorläufigen Mitteilung, die Molodenkow am 16. Dezember 1921 in einer wissenschaftlichen Sitzung der klinischen Konferenz machte, erbrachte er den Beweis, daß alle diese Fälle sicherlich als echte Paranoia aufzufassen seien.

Bei allen diesen Fällen ist es wieder klar zu sehen, daß die Paranoia Kraepelins eine endogen-konstitutionelle Krankheitsform ist mit der Eigenart, daß die Wahnbildung nichts anderes als eine psychologisch verständliche, folgerichtige, unter dem Einflusse äußerer Faktoren sich ausbildende Fortentwicklung der präpsychotischen Persönlichkeit darstellt. Die Vorstellung, die wir daher von der paranoischen Ver- 
anlagung gewinnen, paßt durchaus in den Rahmen der bekannten Schilderung seitens Kraepelin und Bleuler. Das Wesentlichste dieser Veranlagung erblicken wir ebenfalls in einer großen Selbstüberschätzung nebst einer Anzahl intellektueller Unzulänglichkeiten, wie es etwa die Neigungen zu Räsonnements, zu Träumereien und Phantastereien sind.

Die klinische Bearbeitung der Fälle und eine eingehendere psychologische Schilderung der paranoischen Veranlagung überlasse ich dem oben angeführten Autor; ich selbst habe hier die Absicht, einige allgemeinere psychiatrische Fragen in Angriff zu nehmen. Dem Versuch, sich ihrer Lösung zu nähern, diene nun unser Material.

Studiert man die einschlägige Literatur, so muß man sich wirklich wundern: so verwickelt erscheint die ganze Frage, so mannigfaltig und verschiedenartig, ja untereinander unvereinbar sind doch alle die Fälle, die unter dem Namen chronischer Paranoia mit herangezogen werden. Einerseits gibt es hier zweifellos Fälle echter konstitutioneller Paranoia im Sinne Kraepelins, andererseits sind es Beobachtungen, die mit ihr nichts gemein haben, ja unter sich nicht gleichwertig sind. Je mehr man im Komplizierten des Problems Klarheit und Ubersicht gewinnt, desto mehr neigt man zur Úberzeugung, da $ß$ in der ganzen Paranoialehre noch bis jetzt, vielleicht nur unbewußt, die Vorstellung von der Identität der Begriffe der chronischen Paranoia und des Verfolgungswahnes (in weitem Sinne) herrscht. Die Schuld an dieser Identifizierung tragen vielleicht zum Teil Magnans Lehren über den systematisierten Verfolgungswahn. Daß derartige Vermutungen nicht unbegründet sind, beweist allein schon die neueste Literatur. So steht es mit den meisten Lehrbüchern der Psychiatrie, aber auch mit einer ganzen Reihe spezieller Arbeiten über die chronische Paranoia. Fast alle Fälle der Kleistschen Arbeit über die Involutionsparanoia, der Bergerschen Schrift und der Monographie Krïgers - fast alle diese Fälle verlaufen unter den Zustandsbildern des Verfolgungswahns und seiner Unterformen.

Was geben uns nun, von diesem Standpunkt aus betrachtet, die Paranoiafälle, die wir in unserer Klinik beobachtet haben? Wie gesagt, ist die Wahnbildung bei der konstitutionellen Kraepelinschen Paranoia nichts anderes als eine psychologisch verständliche Fortentwicklung der präpsychotischen Persönlichkeit. Die maßlose Selbstüberschätzung, nebst dem Hange zu träumerisch-phantastischer Lebensauffassung, verleitet derartige Individuen auf den Weg großartiger Lebenspläne und Vorsätze, indes ihre geistigen Kräfte keineswegs solchen Aufgaben gewachsen sind; daraus ergibt sich ein Konflikt mit der realen Umgebung, und auf dem Boden des Konfliktes entwickelt sich nun ganz schleichend die Wahnbildung, oder vielmehr, wie Krae- 
pelin es trefflich ausdrückt, entsteht hier jene ,Verrückung“ des Standpunktes gegenüber der Umwelt, wobei eine Art krankhaft. verfälschter Weltanschauung und -auffassung das Endergebnis ist. Einen Jüngling, mit dessen Psyche Kraepelin die Psyche seiner Paranoiker vergleicht, belehren die Mißerfolge: er bildet sich an ihnen zum reifen Manne aus; den minderwertigen Paranoiker treiben sie in den Wahn, wo er nun die Rechtfertigung für sein maßlos gesteigertes Selbstgefühl findet. Ưberall haben wir es hier mit einer Selbstüberschätzung, d. h. mit Größenideen, zu tun; bei diesen konstitutionellen Paranoikern stehen die Größenideen immer im Vordergrunde, während einzelne Ideen des Verfolgtseins und der Beeinträchtigung nur sekundär auftreten, nur als Erklärung dessen, daß ihre Ansprüche nicht anerkannt werden, ihre, wenn man es so sagen darf, „Größe" ignoriert wird. Es ist unzweifelhaft, daß die Verfolgungsideen nicht das Primäre sind: im klinischen Bilde spielen sie durchaus nicht die Hauptrolle, sondern eine nebensächliche und werden überhaupt meistens erst zur Erklärung aller Mißerfolge, denen diese Psychopathen im Lebenskampfe ausgesetzt sind, produziert. Alle diese Behauptungen bestätigen sich mit Anschaulichkeit an der Hand unserer Beobachtungen: wir haben keinen einzigen Fall zu notieren, wo der Verfolgungswahn eine selbständige, im Vordergrunde des Bildes stehende Rolle gespielt hätte; im Gegenteil waren manche unserer Kranken so in sich selbst verliebt, so von dem Gefühl ihrer eigenen Größe durchtränkt, daß sie sich gleichsam über allen menschlichen Händeln und Kleinigkeiten erhaben dünkten. Zur Illustration führe ich nun in kurzer Fassung einige unserer Krankheitsgeschichten an.

a.

1. Pat. E., 23 Jahre alt, Sohn eines Arztes, zur Zeit beim Militär. Eine ganze Roihe Psychopathen mütterlicherseits: anscheinend Pseudologia phantastica in drei Generationen (Urgroßmutter, Großmutter und Tante), parallel schwere Hysterie (bei der zweiten Tante und ihrer Tochter); Vater - mäßiger Alkoholist, ein Vetter - schizophren. Späte Entwicklung, lernt spät Gehen und Sprechen, späte Zahnung; noch mit 8 Jahren ist seine Sprache nur den nächsten Angehörigen verständlich. Bis auf das 4. Lebensjahr höchst unbeweglich und plump, wird er weiter lebhaft und beweglich, ist sehr ängstlich, überhaupt psychopathisch labil, ziemlich ungesellig, aber durchaus ohne moralische Defekte. Auf der Schule leistet er nur Mittelmäßiges, Begabung disharmonisch. Während der Pubertät meint er, seine Eltern Jieben ihn nicht genug, ist ungesellig und zurückgezogen, wird aber etwa mit 16 Jahren in hohem Maße expansiv, hat viele Kameraden und Bekannte: 1917 in der Abiturientenklasse steht er im Brennpunkt eines ganzen Kameradenzirkels, ist Delegierter, vertritt seine ganze Klasse, ist schließlich Präsident des Bureaus des Allrussischen Schülerkongresses (Frühling 1917). Weiter eignet er sich eigenartigs Anschauungen anarcho-individualistischen Gepräges an, die, wie er sich selbst ausdrückt, seinem ,anarchistischen Charakter" durchaus entsprechen, hat aber in der Tat ziemlich konfuse Begriffe von den anarchistischen Lehren. Sein Vater hält ihn für ,,beschränkt", ziemlich ,,interesselos", aber für ,,herzlich gut"; seine Kameraden lieben ihn. Mit 19. Jahren tritt er beim Militär ein: beim Kriegs-Topographie-Ressort, 
im Disnste ist er leistungsfähig, gewandt in Erfüllung mancher Aufträge, wird chlieBlich zum Kommandanten der Behörde ernannt. Im Privatleben orientierte er sioh vorzüglich, war sogar mit der Diamantenbranche wohl bekannt und kostete seinen Eltern keinen Groschen. Stets guter Laune, sich selbst uiberschätzend, hatte er wenig Interesse für Laktüre; meint2, der eigene Kopf sei das beste; hielt sich für ausgezeichnet begabt; äußerte oft die Behauptung, da B er vieles, ja alles könne; all ss müsse ihm gelingen. Im Frühling 1921 verleitet er 10 seiner Alters- und Dienstgenossen zu einem bewaffneten Überfall: etwa 65 Millionen Papierrubel werden einer Munizipalkasse entnommen. Das Geld soll teilweise der Armenunterstützung gedient haben, womit das ganze Verbrechen auch gerechtfertigt wird. Einen ganzen Monat lang war er frei, wurde alsdann auf Anzeige einer 18jährigen Pro* stituierten festgenommen, welche er selbst über das ganze Unternehmen unterrichtet hatte, wobei er auch sie aufforderte, an weiteren ähnlichen Geschäften teilzunehmen. Das Mädchen machte auf ihn einen eigenartigen Eindruck, er hielt sie für ein unschuldiges Opfer übler sozialer Verhältnisse, machte Bekehrungsversuche, schenkte ihr im ganzen ca. 2 Millionen Rubel; außerdem verzechte er in ihrer Gesellschaft noch etwa eine Million Rubel. In der Haft stellte sich bald heraus, da $B$ sein Benehmen und seine Urteile über die Tat durchaus wahnhaft sind; die provisorische Begutachtung und die darauf erfolgte Anstaltsbeobachtung deckten ein Größenideensystem auf, das bereits seit 4 Jahren in Entwicklung begriffon war. Die Wahnbildung schritt in der Haft ziemlich rasch vorwärts und gewann immer schärfere Umrisse. Seit einem Jahr hat er Visionen (hauptsächlich nachts) und ekstatische Zustände. Nun kurz die Wahnideen: er sei ein Mensch, der ganz ausschließlich begabt ist; ein großer Erfinder; er habe ein neues Gesetz, ,das Gesetz von der Wägbarkeit des Gedankens", entdeckt; er besitze die Fähigkeit, Menschen auf besondere Weise zu beeinflussen, unmittelbar und durch Hypnose auf Entfernungen; er habe die Existenz eines zweiten, unsichtbaren, ätherischen Körpers im menschlichen Wesen festgestellt und auf diese Weise endgültig die ewige Unsterblichkeit der Menschheit bewiesen; der Tod sei bloß Übergang in diesen „Zustand der Unsichtbarkeit"; im Vergleiche mit seiner Mission sei Christentum nichts mehr als eine Vorahnung gewesen; die Menschen werden sich zu seinen Lehren bekehren lassin und werden alsdann freiwillig in den Unsichtbarkeitszustand übergehen, d. h. einfach gesprochen, Selbstmord begehen; er selbst für sich beabsichtigt nicht dergleichen bloB seiner Mission wegen, die darin besteht, eine ausführliche Schrift über soine Entdeckungen zu veröffentlichen, Vorträge in russischen Großstädten darüber zu halten usw. Die Tragweite seinər suggestiven Kraft sei so groB, daß er Weltherrscher werden könnte, wenn er es gewünscht hätte; will jedoch bloß durch Überredung wirken, weil ihm alles Gewalttätige verhaßt sei; er gedenkt auch weiterhin, die Menschheit von dem künftigen Untergang zu retten, welcher nach 2 Millionen Jahren wegen der Abkühlung der Sonne statthaben soll; trägt dabei ein Projekt der ,Elektrofikation des Erdballs“ vor, welches auf der Idee beruht, aus der Erdkugel eine kolossale Quelle elektrischer Energie herzustellen: der Erdball soll mit elektrischen Leitungsdrähten umgeben werden, wodurch die Erde zu kolossalem Elektromagnet wird. Eine ganze Reihe Räsonnements produziert der Kranke, indem er hartnäckig zu beweisen bestrebt ist: er habe kein Verbrechen begangen, habe vielmehr zu seiner Tat das volle Recht gehabt.

2. Patient M., 39 Jahre alt, Arzt, Montenegriner. Belastung: Tante väterlicherseits geisteskrank; Epilepsie beim Bruder. Große Langlebigkeit in der Fa. milie. Entwicklungsgang normal, Schulleistungen gut. Von früh auf sehr energisch, selbständig, lebhaft reagierend, pflegte er stets unabänderlich alles durchzusetzen, was er für das eigene und allgemeine Wohl für nützlich und notwendig hielt. Unbequem und schwierig im Verkehr und Zusammenleben, zeichnete er sich 
durçh eine Neigung zu häufigem Ortswechsel und Abenteuem aus. Seine Stimmung ist stets ,prachtvoll", er ist lebenslustig, jedoch etwas reizbar. Sein ganzes Leben, bis auf die jüngste Zeit, ist eine kontinuierliche Reihe von Abenteuern und Konflikten mit der Umgebung, was als Folge der fanatischen Durchsetzung seincr Lebensanschauungen anzusehen ist. Stets ist er am Werke, seinen Ideen möglichst weite Verbreitung zu verleihen, nimmt aber dabei sehr wenig Rücksicht auf seine Mitmenschen. Seine Weltanschauung besteht hauptsächlich in folgendem: er ist Kommunist. Freund des Fortschrittes und Feind der Routine auf allen Gebieten; allen Menschen, mit denen er zu tun hat, wirft er vor, daß sie rückständig und träge seien und beruflich nichts taugen; als Arzt preist er alles Neue in der Medizin, sucht absolut originelle Wege in der Behandlung verschiedener Krankheiten: die Wege sollen auf einem tieferen und ernsteren Verständnis der Natur im allgemeinen, der menschlichen Natur im besonderen, fußen; praktisch sind seine Heilmethoden absonderlich, manchmal riskiert und unvorsichtig: Bronchitis wird z. B. mittels Inhalationen beliebiger feinkörniger Pulverstoffe, etwa Kalk, Gips, ja sogar Erde behandelt usw. Eine große Reihe Reformen auf dem Gebiete der Ernährung wird vorgeschlagen, in der Voraussetzung, da $B$ die Zahl der tauglichen Nahrungsobjekte in hohem Maße erweitert werden kann: es seien z. B. Gras, Lehm, Kohle usw. ge'nießbar. „Ein überzeugter Materialist" in seinen ,wissenschaftlichen" Anschauungen, trägt er seine Ideen in einer gedrängten, rein formellen Weise vor; der In: begriff seiner Anschauungen und seiner Methode, die Natur zu behandeln, lassen sich recht wohl zusammenfassen in der Formel ,ortparampoli“, einer Abkürzung für die Kombination der Wörter: orto, para, meta und poli; er bediene sich in bezug äuf alle Dinge der Welt verschiedener Betrachtungsweisen: es existieren nämlich eine „Orto-Methode“" eine „Para-Methode“" usw.; was das alles zu bedeuten hat, darüber ergeht sich Pat. in ziemlich unklaren, rein formellen, räsonierenden Aus. lassungen. Der Sinn all dieser Räsonnements ist, wie es scheint, darin zu suchen, daß der Kranke sämtliches dem Menschenverstande zugänglich $€$ s Wissen, alle Wissenschaften in einem knapp gehaltenen, gedrängten Ganzen zu systematisieren und $\mathrm{zu}$ vereinheitlichen nach seiner Art bemüht ist. Fanatisch bestrebt, seine Ansichten zu verbreiten, sucht er überall Vorwände dazu, gerät dabei beständig in Konflikt, hält nirgends lange aus, muß immer disputieren und sieht natürlicherweise in jedem und allem entweder elende, zurückgebliebene Menschen oder gar Feinde. Dessenungeachtet geht es ihm stets ,ausgezeichnet", seine Stimmung ist durchweg gut, seine Unternehmungslust groß: nichts hält ihn ab von der Verwirklichung seiner absurden Ideen. Trotz seinen Wahnkonstruktionen bekleidete Pat. verantwortungsvolle Amtsstellungen als Arzt und hatte eine Reihe wichtiger An. gelegenheiten im Auftrage der Kommunistischen Partei, deren Mitglied er ist, zu erledigen.

3. Patientin G., 45 Jahre alt. Heredität: Vater und Mutter-mäßige Alkoholisten, desgleichen die Brüder der Pat. Über ihre ersten Kindheitsjahre fehlt jegliche Nachricht. Mit 21 Jahren verheiratet, hatte sie 14 mal Wochenbett, eine Fehlgeburt und wurde mit 42 Jahren Witwe. Lebte stets in einer Provinzstadt wo sie sich mit Markthandel beschäftigte. Von Kindheit an religiös, nahm sie an Pilgerwanderungen nach Klöstern teil. "Schon als Kind“ wußte sie, „daß es Teufel gibt." Mit 12 Jahren erlernte sie selbständig das Lesen und las mit Eifer das Heilige Testament, welche Lektüre auf sie großen Eindruck machte; schon seit ihrer Jugend hat sie viel über religiöse Themata nachgedacht. Mit 14 Jahren lernte sie Leute aus der Baptistensekte kennen, mit denen sie ihre Beziehungen heimlich vor den Eltern weiterpflegte. Nach der Heirat mußte sie, durch äußere Umstände, Schwierigkeiten und pekuniäre Sorgen gezwungen, ihre Beschäftigung mit den religiösen Fragen einstweilen aufgeben, um erst wicder im Alter von 
35 Jahrer ein Neuerwachen ihres religiösen Lebens zu erfahren. Nun betet sie viel, fastet, kämpft mit den Sünden, d. h. ,,mit dem Teufel“", hält jedoch ihre religiösen Erlebnisse vor der Umgebung geheim. Nach einer Vision (sie sah ein feuriges Kreuz) erlebt sie eine Reihe Offenbarungen religiösen Inbalts. Einst sieht sie, wie die Gottesmutter das Heiligenbild verließ, sich ihr näherte, sie segnete und ihr zugleich ein Schwert darbot. Als Folge entstand ein Wahnsystem, das mit dem Einschlagen der Revolution sich besonders üppig entfaltete; damals versuchte die Pat. Reden in öffentlichen Versammlungen zu halten. Sie war gegen die moderne Geistlichkeit gestimmt; behauptete, daß dieselbe vom Teufel ihr Amt erhalten habe, klagte sie aller Sünden an und haßte besonders Rasputin und den Patriarchen; der Heilige Geist habe sie (Pat.) aufgesucht, sie sei zu einer großen Mission erkoren, die Kirche Christi auf Erden soll sie erneuern; die Gottesmutter selbst habe ihr dazu den Segen erteilt, das Schwert zu diesem Zwecke dargeboten. Als Folge: Mordversuch am Patriarchen, Festnahme durch die Obrigkeit und ärztliche Beobachtung in der psychiatrischen Klinik. Pat. hält sich für vollkommen gesund und erklärt ihre Handlungsweise für richtig; unerschütterliches Wahnsystem; Auftreten demütig, aber nicht ohne Würde.

4. Patient Tch., 28 Jahre alt, berufsmäBiger Chiromant. Bildungsgrad: Volksschule. Heredität: Mutter geisteskrank, Bruder - Alkoholist. Stammt aus einer Bauernfamilie; entwickelte sich normal und war von Kindheit an lebhaft, heiter, gesellig, gutmüitig, dabei hartnäckig und fanatisch in seinen Überzeugungen. Mit 14 Jahren fängt er sein selbständiges Leben an, wechselt öfters den Beruf und macht schließlich, als Matrose, einen Abstecher in Baku, wo er zufällig einen Chiromanten kennen lernt, dessen Wahrsagungen auf ihn einen großen Eindruck machen: er wird sein Schüler und schließlich ist auch er berufsmäßiger Wahrsager. Eine Neigung zu Geheimnisvollem und Mystischem hatte er schon vorher. Mit 15 Jahren erstmalig Gesichtstäuschungen: er sieht den Tod seines Vaters in allen Details, trotz der Entfernung von mehreren tausend Kilometern. Von diesem Augenblick an meint der Kranke, befinde er sich in ,engen Beziehungen zu der Welt des Jenseits"; hier setzt die Wahnbildung an, die später sein ganzes Leben bestimmt und beherrscht. Unter dem Einflusse der Wahnideen beginnt der Kranke mit 20 Jahren eine Wohltätigkeitsaktivität großen Stils. Allmählich setzt sich ein stabiles Wahnsystem fest, dessen Inhalt, wie folgt, zusammengefaßt werden kann: er ist ein ganz besonderer Mensch; er besitzt die Fähigkeit eines freien Verkehrs mit der Geisterwelt, es ist ihm möglich, das Weltall und speziell das Menschenleben von einem Standorte aus zu betrachten, welcher sonst den Sterblichen vollkommen unzugänglich ist; die Vergangenheit und die Zukunft liegen ihm offen; trotz der Entfernung kann er auf Menschen Einfluß üben und mit ihren Seelen unmittelbar verkehren; selbst mit dem höchsten Wesen, d. h. mit Gott, befindet er sich im ständigen Verkehr; Gott habe ihm befohlen, eine Wohltätigkeitsarbeit zu entwickeln, insbesondere auf dem Gebiete der Kinderfürsorge, da in den Kindern die Zukunft der Menschheit liege. Als Chiromant genießt Pat. großen Ruf, lebt pekuniär ausgezeichnet, gibt den größten Teil seiner Einnahmen für Einrichtung von Kinderheimen, Speisehäusern für Kinder usw. aus, 1918 gründet er den „Brüderbund“ - eine Gesellschaft, die sich das Ziel setzt, arme Kinder und Kriegsgefangene möglichst ausgiebig zu unterstützen. In Moskau organisiert Pat. eine Anzahl Kinderspeisehäuser und reist 1919 nach der Ukraine zwecks Zuckerankaufs; es gelingt ihm auch, eine stattliche Zuckerpartie nach Moskau zu befördern, aber, an Ort und Stelle angelangt, erfährt er, daß sein Zuckervorrat zugunsten der gesamten Bevölkerung der Stadt Moskau konfisziert werden soll. Pat. will es nicht zulassen, verkauft den ganzen Vorrat zu einem Schieberpreis und reist mit dem Gelde nach der Stadt Jekaterinburg, um daselbst Kinderheime zu organi- 
sieren. In Jekaterinburg bekommt er eine Nachricht aus dem Jenseits, daß ihm große Unannehmlichkeiten bevorstehen, die jedoch ein günstiges Ende nehmen werden. In der Annahme, daß diese Unannehmlichkeiten im direkten Zusammenhange mit der Zuckerspekulation stehen, meint Pat., daß ihr Eintreten möglichst beschleunigt werden möge, und meldet sich deshalb selbst bei der Außerordentlichen Kommission (der sogenannten "Tscheka"); zugleich erstattet er das ganze übrige Gald, nebst einer exakten Rechenschaft über die verausgabten Summen, zurück. Pat. wird sofort festgenommen, die Angelegenheit bekommt ernste Wendung, es droht ihm Todesstrafe - jedoch zu 20 Jahren Haft verurteilt, wird er nach Moskau gebracht und gerät hier zum erstenmal in seinem Leben in den Bannkreis der Psychiater. Pat. wird anstaltsmäßig beobachtet und, als Geisteskranker, von der Strafe freigesprochen. In der Klinik ist er ständig in guter Stimmung, rüstig, sehr gesellig. Hält sich für vollkommen gesund und verhält sich mit überlegenem Bedauern allen gegenüber, die seine Wahnideen nicht als Wahrheit gelten lassen wollen. Mit unerschütterlicher Überzeugung spricht er darüber, redet von seiner Exklusivität: ihm sei die Erkenntnis aller Dinge möglich, weswegen er auch keine Lektüre treibt, da er im voraus genau den Inhalt der Bücher kenne. Halluziniert, verkehrt mit dem ,höchsten Wesen“ und mit den Geistern verschiedener verstorbener Personen; versinkt dabei in einen ungewöhnlich erhabenen und angenehmen, anderen unzugänglichen, ekstatischen Zustand. Ist körperlich gesund: jegliche Krankheitszeichen seitens des Nervensystems fehlen.

Wie paradox es auch anmuten kann, scheint es, daß an der Hand unserer Fälle wir notwendigerwcise zu der Folgerung gelangen müssen, daß ein Verfolgungswahn, wohl systematisiert und im Vordergrunde des klinischen Bildes stehend, keinesfalls für die in Rede stehende Krankheitsform kennzeichnend ist. Zu dieser Krankheitsform gehört cine Reihe konstitutioneller Psychopathen, vielleicht auch nicht vollständig gleichartiger Prägung, immerhin durch eine Anzahl bestimmter oben erwähnter Zeichen charakterisiert, die nicht:s damit zu tun haben, was seitens anderer Autoren als chronische Paranoia mit Verfolgungsideen beschrieben wird. Sämtliche Fälle, wo die Verfolgungsideen im weitesten Sinne des Wortes primär sind und das ganze Bild beherrschen, sämtliche Fälle dieser Art sollten demnach streng von der ,,echten" Paranoia unterschieden werden; und es wäre am besten, diese zwei Bagriffe auch mittels verschiedener Bezeichnungen wohl auseinander zu halten.

Doch ist die Gruppe der echten Paranoia seitens Kraepelin so meisterhaft-künstlerisch zusammengefaßt worden, daß ihr gewiß der alte Name - die Paranoia Kraepelins - allein gebührt, obgleich der Ausdruck ,Megalomania paralogica“ vielleicht am treffendsten wäre. Hierher gehören hauptsächlich: Erfinder, Wahrsager, Heilkünstler, Prediger aller Art, Leute mit verschiedenen Neuerunsgplänen - meist ziemlich harmlose Personen, die, dem Wahne zum Trotz, leidlich gut sich mit dem realen Leben abzufinden imstande sind. Mit der Bezeichnung Megalomania will ich keineswegs sagen, daß alle diese Fälle zur chronischen Manie, etwa im Sinne Spechtscher Anschauungen, zu zählen seien; im Gegenteil meine ich, daß man der paranoischen Ver- 
anlagung durchaus eine selbständige Existenz zuschreiben soll; wie jede psychopathische Veranlagung, ist sie anderen Veranlagungen sehr nahe verwandt, was sie aber keinesfalls ihrer Selbständigkeitsrechte beraubt. Die Verwandtschaft mit der cyclothymen Veranlagung einerseits gibt sich manchmal durch eine intensivere Affektfärbung kund, während andererseits die intellektuellen Besonderheiten - der Hang zu Räsonnements und insbesondere die Neigungen zu Träumereien und zum Phantasieren - die paranoische Veranlagung als der hysterischen besonders nahe verwandt erscheinen lassen. In mehreren von uns beobachteten Fällen sahen wir ein erhöhtes Selbstgefühl in deutlicher Ausprägung, und doch konnte von chronischer Manie nicht die Rede sein, da keine stärkere Affektstörung vorlag, die übrigen Maniesymptome fehlten, während auf dem Verstandesgebiete sich deutlich jene eben genannten Besonderheiten abhoben, die kaum, doch in ähnlichem Maße manischen Zuständen eigen sind.

Akute und chronische Erkrankungen, bei denen der Verfolgungswahn den Hauptkern des Krankheitsbildes ausmacht und oft nicht als psychologisch verständliche Entwicklungslinie der Gesamtpersönlichkeit des Kranken zu deuten ist, sollte man überhaupt aus der Paranoiagruppe ausscheiden: solche Fälle, die nach eingehender Untersuchung sich sehr wohl anderweitig einordnen lassen, sollten auch anderweitig und unter anderen Bezeichnungen beschrieben werden.

Besteht nun für uns kein Zweifel darüber, daß die Paranoia im obigen Sinne sich auf dem Boden einer besonderen Veranlagung entwickelt, so läßt sich wohl fragen: Was ist nun der Boden, welcher akute und chronische Verfolgungswahnfälle zeitigt, die, wie gesagt, mit der paranoischen Veranlagung kaum etwas Gemeinsames besitzen?

Die Antwort darauf wäre die, daß solche Fälle meist auf Vergiftungen exogener und endogener Art beruhen: es gehören hierher akute und chronische paranoide Fälle bei chronischem Alkohol- und Cocainmißbrauch, bei Hirnlues, Schizophrenie und Epilepsie, endlich auch bei Involutionsprozessen, d. h. bei Psychosen des Rückbildungsalters und bei cerebraler Arteriosklerose. In diesen Fällen haben wir es mit einer ganzen Reihe sogenannter krankhafter Prozesse - mit organischen Geisteskrankheiten in weitem Sinne - zu tun. Es gibt hier immer eine ziemlich greifbare Ursache, auch einen Krankheitsanfang, wobei die Gesamtkrankheit und der Wahn sich immer, als etwas Fremdartiges und Neues, der Psyche des Patienten aufdrängen. Die Frage, warum hier eben Wahnbildungserscheinungen zustande kommen und nicht etwa andere Zustandsbilder, läßt sich nur in derselben Richtung beantworten, wie auch in bezug auf andere organische Krankheiten ähnliche Fragen beantwortet werden (ähnlich der Frage z. B.. wie bei der Paralyse die verschiedensten psychotischen Zustände möglich 
sind); es gehört diese Frage zu den großen Zeitfragen der Psychiatrie und erfordert spezielle Forschungen und Studien. Auch ist bis jetzt noch unklar, ob hier nicht gewisse konstitutionelle seelische Eigentümlichkeiten des Kranken eine Rolle spielen oder etwa umgekehrt Faktoren wie das Alter, das Milieu und andere Exogenien. In diesem Artikel aber interessieren uns nicht eigentlich diese Erkrankungen, sondern vielmehr Verfolgungswahnfälle, die auf rein konstitutionellem Boden entstehen.

Zweifellos gibt es solche Fälle: akute und chronische, die rein konstitutionell bedingt sind. Was für Veranlagungen könnten es sein, die hier den Boden abgeben? Die paranoische Veranlagung im Sinne echter Kraepelinscher Paranoia dürfte, wie oben ausgeführt, kaum für diese Fälle verantwortlich gemacht werden. Es läßt sich demgemäß die Frage aufstellen, ob es nicht vielleicht eine besondere Veranlagung gebe, auf deren Boden derartige akute und chronische Erkrankungen mit Verfolgungsideen zur Entwicklung gelangen? Die Frage muß vorläufig unbeantwortet bleiben, doch aber möchte man glauben, daß vielleicht auch kein Bedürfnis vorliegt, um den Begriff einer derartigen besonderen Veranlagung aufstellen zu wollen. Denn es reichen hier unsere alten wohlbekannten Veranlagungsbegriffe vollkommen aus und ermöglichen uns eine durchaus befriedigende Erklärung für die Frage nach dem Boden, auf dem sich solche paranoide Zustände aufbauen.

Gedenken wir nun der bekannten Kleistschen Einteilung sämtlicher psychopathischer Veranlagungen in autochthon-labile und reaktiv-labile. Nun dürfen keine Zweifel bestehen, daß Verfolgungswahnideen autochthoner Art auf dem Boden cyclothymer Veranlagung entstehen können, und zwar wie auf dem Boden der chronischen Erregung, so auch auf dem der chronischen Verstimmung. Ist man geneigt, die chronische konstitutionelle Verstimmung von der psychasthenischen Verenlagung abzutrennen und diese letzte als eine selbständige Größe aufzufassen, so muß behauptet werden, daß auch auf dem Boden der Psychasthenie unser Symptomenkomplex seine Ausbildung finden kann. Jedoch wohl am häufigsten kommen hier manisch-depressive Mischzustände in Betracht, ein Umstand, in dem vielleicht die Ursache des Unterfangens zu suchen wäre, welches die chronische Paranoia mit dem m.-d. Irresein zu identifizieren sich bemühte. Es gibt gewiß manisch-depressive Fälle, bei denen die Anfälle jedesmal in der Gestalt paranoider Zustandsbilder auftreten, was ja auch den Anlaß gab, eine periodische Paranoia abzutrennen. Solche Fälle gestatten die Annahme, daß es wirklich geboten ist, unter den Mischzuständen des m.-d. Irreseins gewisse Unterarten unterscheiden zu lernen, bei denen eine größere Neigung zu Wahnkonstruktionen besteht. Nur in diesem Sinne hat vielleicht Ewald recht, indem er unter dem Namen der 
Hyponoia eine besondere Unterart cyclothymer Veranlagung abgrenzt; sicherlich hat er aber unrecht, wenn er die selbständige Existenz der echten Paranoia in Abrede stellen will.

Weiterhin kann man annehmen, daß es autochthone akut-paranoide Eisisoden auch auf dem Boden schizophrener Veranlagung (Gannuschkin) oder bei schizoiden Persönlichkeiten (Kretschmer) geben kann. 1914 äußerte Gannuschkin die Annahme, daß die der Schizophrenie, als einem Krankheitsprozeß eigenen, so meisterhaft durch Kraepelin, Bleuler und Stransky aufgedeckten seelischen Mechanismen vielleicht auch der normalen Psyche der Gesundheitsbreite innewohnen; auf Grund dieser Erwägung und einer Reihe anderer läßt er die Existenzmöglichkeit einer schizophrenen Veranlagung zu. Die endgültige Anerkennung einer solchen Veranlagung und ihre Einreihung in die Nähe anderer psychopathischer Veranlagungen erscheint uns an der Hand klinischer Tatsachen und eines eingehenderen Studiums abnormer Charaktere immer mehr und mehr als dringend und geboten. Beiläufig gesagt, sollten von diesem Standpunkte aus die Fragen von den von Kraepelin beschriebenen paranoiden Episoden bei den Verschrobenen und ihrer Verwandtschaft mit den abortiven Paranoiaformen ihre Revision finden.

Was nun die reaktiven Irreseinsformen, die unter dem Bilde des Verfolgungswahnes verlaufen, anbetrifft, so liegen ihnen die verschiedensten Veranlagungen zugrunde.

Hier kommen erstens in Betracht die eben genannten zwei Veranlagungen: die cyclothyme und die schizophrene. Weiter spielen hier eine große Rolle die hysterische Veranlagung und eine Reihe ihr verwandter abnormer Charaktere: Lügner und Schwindler, Haltlose, Affektiv - Epileptische oder Triebmenschen. Dafür spricht eine ganze Reihe reaktiver Erkrankungen, die in der Haft beobachtet wurden. In diesem Sinne hat vielleicht Wilmanns recht, wenn er behauptet, daß die Paranoia nichts anderes sei als eine krankhafte Verirrung verschiedener Psychopathen; nichts als eine durch äußere Umstände bedingte Entgleisung in der Richtung des Wahnes; er hat aber sicherlich unrecht, wenn er die Kraepelinsche Paranoia in diesen Psychopathien auflöst, ihre selbständige Existenz in Abrede stellt.

Alle diese Behauptungen beziehen sich gewiß mehr auf die akuten Formen des Verfolgungswahnes - wie kommen wir nun am besten zum Verständnis der Genese seiner chronischen Formen?

Hier ist es vielleicht angebracht, einiges über den sogenannten paranoischen Charakter zu sagen, d. h. über Leute, denen ein besonderer Argwohn innewohnt, die reizbar und verschlossen sind, leicht kränkbar und eigensinnig; über Leute, die überall und in allem etwas auf sie Bezogenes, sie unmittelbar Betreffendes, wähnen. Im Sinne obiger 
Erörterungen ist es kaum möglich, diese Charakterart der Kraepelinschen Paranoia und der paranoischen Veranlagung im obigen Sinne beizurechnen; und, mag es auch auf den ersten Blick seltsam scheinen, es ist doch vielleicht richtiger, diese Charaktervariante anderen Veranlagungen zuzuzählen, auf deren Boden, wie oben ausgeführt, der Verfolgungswahn seine Entwicklung findet. Wir lassen vorläufig die Frage offen, ob die hysterische psychopathische Konstitution hier mitverantwortlich gemacht werden könnte, desgleichen auch die psychasthenische, falls ihr selbständige Existenz gebührt, und wenden uns zu der cyclothymen und schizophrenen Veranlagung. Und nun glauben wir mit genügender Sicherheit behaupten zu dürfen, daß ein gewisser Bruchteil dieser Charaktergruppe, wenn auch vielleicht ein geringerer, der cyclothymen Veranlagung zuzuschreiben ist; ein anderer, größerer Teil - der schizophrenen. Diejenigen Charaktere, bei welchen im Vordergrunde die Züge der Verschlossenheit, des Eigensinns, einer Unzugänglichkeit im weiten Sinne stehen, bei denen eine schärfer ausgeprägte Fähigkeit, überall eine gewisse Eigenbeziehung aufdecken zu müssen, vorhanden ist - solche Charaktere sollte man eher der schizophrenen als der paranoischen Veranlagung beirechnen; andere wieder, wo als Grundzüge Argwohn, Reizbarkeit, Heftigkeit, d. h. eine Reihe komplizierter Mischaffekte, ohne Unzugänglichkeit und Verschlossenheit, vorwalten, wäre es vielleicht richtiger, für die cyclothyme Veranlagung zu reservieren.

Lassen wir nun gelten, daß auf dem Boden einer chronischen, d. h. dem betreffenden Individuum innewohnenden im Kerne seines pathologischen Charakters liegenden cyclothymen Veranlagung besonderer Unterart (Ewald), insbesondere bei alternden Leuten, ein chronischparanoider Zustand sich ausbilden kann, so liegt doch die Frage auf der Hand: ob auch nicht der schizophrene Charakter, seine Existenz zugegeben, bei ungünstigen äußeren Anlässen für die Entstehung solcher chronisch-paranoiden Bilder einen günstigen Boden darstellt? Vielleicht muß eben in dieser Richtung die Lösung der Frage von der Genese einiger chronischen Verfolgungswahnformen gesucht werden.

Eine Stellung für sich könnten nun auch diejenigen Fälle beanspruchen, die Stransky unter dem Namen der ,Uberwertigen“" zusammenfaßt, denn wir haben es hier wiederum mit einer besonderen Veranlagungsvariante $\mathrm{zu}$ tun: es sind Leute, deren Gedankenleben unter dem Zeichen affektbetonter Vorstellungskomplexe steht. Äußere Einflüsse und Einwirkungen spielen hier eine sehr große Rolle, so daß man diese Gruppe, mit vollem Recht, unter die reaktiven Formen zählen kann. Hierher gehören manche Querulanten, vielleicht auch die seltenen Fälle von Eifersuchtswahn. Die Verschiedenartigkeit des Verlaufs dieser Fälle könnte, mutmaßlich, darin begründet liegen, ob 
und inwieweit die exogenen auslösenden Faktoren ausgeschaltet sein können. Zuletzt wenden wir uns zu Gaupps ,abortiven Paranoiafällen und zu den Friedmannschen ,milden Paranoiaformen". Die Frage, ob sie hierher gehören oder nicht, müssen wir offen lassen. Jedoch ist es dringend zu raten, beim Studieren ähnlicher Fälle besonders eingehend die Charakterart im Auge zu haben, wiewohl auch jedesmal die Frage zu erwägen, ob hier dem Krankheitsbilde nicht eine von den oben besprochenen Veranlagungen zugrunde liegt. Gaupps Fälle scheinen uns, in diesem Zusammenhange, der konstitutionellen Verstimmung nahe verwandt zu sein. Die Ausschaltungsmöglichkeit resp. Unmöglichkeit äußerer Noxen kann hier gewiß für den Verlauf von Belang sein, wie überhaupt bei allen reaktiven Psychosen.

Fassen wir nun zum Schlusse alles Gesagte zusammen, so lauten unsere Ergebnisse folgendermaßen: Es kann sein, daß es in der Lehre von der Paranoia und den paranoiden Veranlagungen richtiger und fruchtbringender wäre, den Standpunkt einzunehmen, daß die echte Paranoia Kraepelins existiert und sich aus einer bestimmten paranoischen Veranlagung entwickelt, welche zu denjenigen Formen, die, das klinische Bild des Verfolgungswahns bietend, früher im Rahmen akuter und chronischer Paranoia beschrieben wurden, gar keine Beziehungen aufweist; es ist sehr wohl möglich, daß diesen letzteren Formen eine ganze Reihe anderer psychopathischer Veranlagungen zugrunde liegt.

Und endlich: Mit der Behauptung, daß der paranoische Wahn im Sinne Kraepelins nichts anderes als eine folgerichtige psychologisch verständliche, aus den Konflikten mit dem realen Leben sich ergebende Entwicklung einer bestimmten Charakterart ist, fällt eine Anzahl Fragen weg, die sonst für den Psychiater so wichtig schienen wie etwa die Fragen nach dem Alter der Patienten, nach den Sinnestäuschungen u. dgl.

Die Krankheit kann im beliebigen Alter einsetzen, hängt doch ihr Ausbruch eben von zwei Komponenten ab: von der Veranlagung und von den Noxen der Umgebung, wiewohl auch von dem Quantitativen beider Faktoren - dem Maße, in welchem die paranoide Veranlagung ausgeprägt ist auf einer Seite und der In- und Extensität der äußeren Einwirkungen auf anderer. Einer von unseren Kranken war 28 Jahre alt, ein anderer gar $23 \mathrm{Jahre,} \mathrm{welcher} \mathrm{Umstand} \mathrm{uns} \mathrm{gar} \mathrm{nicht} \mathrm{zu} \mathrm{wun.}$ dern braucht, wenn wir alle die Perturbationen unseres jetzigen Lebens nicht außer acht lassen.

Sinnestäuschungen kommen bei der echten Paranoia vor, stehen jedoch im innigen Zusammenhange mit dem Wahnsystem, tragen häufiger das Gepräge psychischer Halluzinationen und werden von den Kranken nicht selten in besonderen ekstatischen Zuständen erlebt. 
Und letztens liegt die Vermutung nahe, da $B$ akute, $d . h$. heilbare, psychotische Episoden kaum irgendwie aus der paranoischen Veranlagung sich entwickeln können: die Art, wie hier die Wahnideen produziert werden, der ganze Entstehungsmechanismus des Wahnsystems scheint schon a priori die Annahme dieser Möglichkeit auszuschließen. Und in der Tat hatten wir es in allen unseren Fällen mit Wahnideen zu tun, welche, wenn auch in schubweiser Entwicklung begriffen, außerordentlich hartnäckig und durchweg unkorrigierbar waren.

8. I. 1922.

\section{Literaturverzeichnis.}

1) Kraepelin, Psychiatrie. 8. Aufl. 1915. - $\left.{ }^{2}\right)$ Bleuler, Psychiatrie 1920.

3) Gannuschkin, P. B., Das räsonnierende Irresein. (Korssakoffsche Zeitschr. 1905 [russisch].) - 4) Kleist, Die Involutionsparanoia. Allg. Zeitschr. f. Psychiatr. u. psych.-gerichtl. Med. 73. 1913. — 5) Berger, Klinische Beiträge zur Paranoiafrage. Monatsschr. f. Psychiatr. u. Neurol, 34. 1913. — ${ }^{6}$ Krüger, Die Paranoia. Springer. Berlin 1917. - 7) Specht, Über die klinische Kardinalfrage der Paranoia. Zentralbl. f. Nervenheilk. u. Psychiatr. 1908. - ${ }^{8}$ ) Kleist, Die Streitfrage der akuten Paranoia. Zeitschr. f. d. ges. Neurol. und Psychiatr. Orig. 5. 1911. - $\left.{ }^{9}\right)$ Kretschmer, Wahnbildung und manisch-depressiver Symptomenkomplex. Allg. Zeitschr. f. Psychiatr. u. psych.-gerichtl. Med. r1. 1914. - ${ }^{10}$ ) Ewald, Paranoia und manisch-depressives Irresein. Zeitschr. f. d. ges. Neurol. u. Psychiatr. Orig. 49. 1919. - 11) Gannuschkin, $P$. B., Über die schizophrene Veranlagung, eine Fragestellung (Moderne Psychiatrie 1919 [russisch]). - 12) Kretschmer, Körperbau und Charakter. Springer, Borlin 1920. - ${ }^{13}$ ) Wilmans, Zur klinischen Stellung der Paranoia. Zentralbl. f. Nervenheilk. u. Psychiatr. 33. 1910. - 14) Stransky, E., Lehrbuch der allgemeinen und speziellen Psychiatrie. Leipzig 1919. — 15) Friedmann. Beitrag zur Lehre von der Paranoia. Monatsschr. f. Psychiatr. u. Neurol. 27. 467. - 16) Gaupp, Über paranoische Veranlagung und abortive Paranoia. Neurol. Centralbl. 28. 1909. -17) Meyer, Beiträge zur Kenntnis des Eifersuchtswahns mit Bemerkungen zur Paranoiafrage. Arch. f. Psychiatr. u. Nervenheilk. 46, H. 3. 